\title{
Integration von Kryptowährungen in das Angebot von Regionalbanken
}

\author{
Michael Mathys und Raul Gimeno
}

\section{Zusammenfassung}

Der vorliegende Beitrag untersucht die Einführung von Kryptowährungen bei Regionalbanken. Im Fokus liegt die Einführung der Kryptowährungen, wobei mit der zugrunde liegenden Blockchain-Technologie weitere Anwendungsmöglichkeiten denkbar sind, welche hier nicht berücksichtigt werden. Die folgenden Dienstleistungen wurden identifiziert, welche auf Basis von Kryptowährungen angeboten werden können: (1) Kryptowährung als Asset: Die verschiedenen Kryptowährungen können zur Wertanlage dienen; (2) Kryptowährung als Zahlungsmittel: Der originäre Zweck der Kryptowährung, digitaler Austausch zum Bezug von Waren und Dienstleistungen; (3) Begleitung von Initial Coin Offering (ICO): Kapitalbeschaffung für kleine und mittelgroße Unternehmen; (4) Kredite in Kryptowährungen: Finanzierungen in digitalen Währungen für Kunden mit Erträgen in Kryptowährungen. Bis auf die Begleitung von ICO bedingen sämtliche Umsetzungen technische Anpassungen an der aktuell eingesetzten Software unserer Modellbank. Neben den Anpassungen der Modellbank bietet sich bei allen möglichen Dienstleistungsangeboten eine Zusammenarbeit mit auf Kryptowährungen spezialisierten Unternehmen an. Neben der schnelleren Implementierung aufgrund eines Einkaufs in bestehende Lösungen kann so auch das Entwicklungsrisiko reduziert werden und es muss intern weniger Know-how aufgebaut werden.

M. Mathys ( $\square)$

Worb, Schweiz

E-Mail: michael.mathys@mmhu.ch

R. Gimeno

Berner Fachhochschule, Bern, Schweiz

E-Mail: rauldiego.gimeno@bfh.ch

(C) Der/die Autor(en) 2021 


\subsection{Einleitung}

Die Blockchain-Technologie und die darauf aufbauenden Kryptowährungen sind Themen, welche die Wirtschaft bewegen. Aktuell fehlt ein Grundlagenpapier, um die Konsequenzen und Auswirkungen der neuen Technologien auf Regionalbanken abschätzen zu können. Das Ziel dieses Beitrags besteht darin, eine Einschätzung der Folgen einer Einführung von Kryptowährungen bei Regionalbanken und die notwendigen Änderungen zur Implementation zu beschreiben. Anhand unserer Modellbank, welche eine typische Regionalbank repräsentiert, werden wir die Handlungsfelder für die Integration von Kryptowährungen diskutieren. Mit der Verbreitung der Kryptowährungen müssen sich auch die Banken mit den entsprechenden Konsequenzen beschäftigen. Gerade als Zahlungsverkehrsdienstleister sowie als Anlageberater sind die Banken direkt betroffen. Auch die Kreditvergabe, das Hauptgeschäft der Regionalbanken, könnte durch die Blockchain-Technologie und Kryptowährungen tangiert werden (McMillan 2018).

\subsection{Forschungsfragen}

Mit diesem Beitrag sollen die Grundlagen für die strategische Diskussion um Kryptowährungen bei Regionalbanken geschaffen werden. Folgende Forschungsfragen in Bezug auf Regionalbanken werden behandelt:

- Welche Bankenbereiche sind von Änderungen aufgrund der Kryptowährungen betroffen?

- Welche Anpassungen werden mit der Verbreitung von Kryptowährungen in der Produktpalette notwendig?

- Welche unmittelbaren Handlungsoptionen bestehen?

Abb. 7.1 stellt eine Übersicht über die verschiedenen Fachbereiche einer Modellbank dar, wobei nur die wichtigsten Themengebiete der jeweiligen Fachgebiete ausgewiesen sind. Die verschiedenen Fachbereiche werden im Abschn. 7.8 zusammen mit den möglichen Adoptionen kurz beschrieben.

Nach Erläuterung wichtiger Begriffe und Anwendungsmöglichkeiten von Kryptowährungen werden mehrere Umsetzungsszenarien von Kryptowährungen bei Regionalbanken erarbeitet. Basierend auf Abb. 7.1 wird für jeden Fachbereich der entsprechende Anpassungsbedarf an Software und Prozesse ermittelt. 


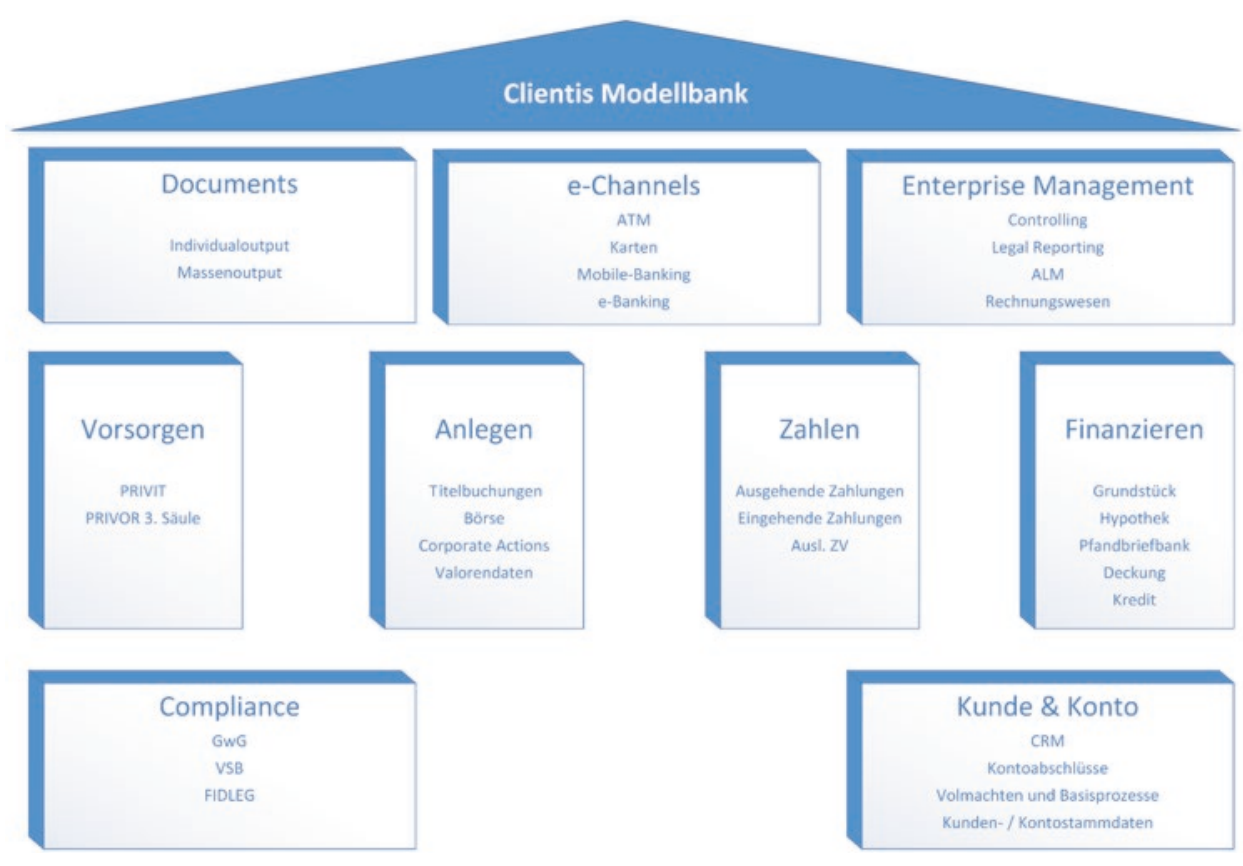

Abb. 7.1 Fachbereiche einer Modellbank

\subsection{Die Token}

Ein digitales Token ist eine Art Coupon, der einen Anteil an einem Projekt bezeugt. Im Bereich der Blockchain-Technologie werden unterschiedliche Token-Kategorien genannt, welche sich je nach Quellen in ihrer Definition und Anzahl unterscheiden. Im Weiteren werden nur Zahlungs- und Anlage-Token kurz erklärt (Bieri und Uhlmann 2019; FINMA 2018; Gussmann 2018)

Zahlungs-Token entsprechen der allgemein bekannten Kryptowährung. Diese sind dazu gedacht, als Zahlungsmittel für Waren und Dienstleistungen eingesetzt zu werden. Die Kryptowährungen vermitteln keine Ansprüche gegenüber einer bestimmten Person (kein Emittent). Auf Basis dieser Tokens lassen sich weitere Applikationen aufbauen. Diese Token fallen unter das Geldwäschereigesetz.

Anlage-Token repräsentieren Vermögenswerte. So können Anteile an zukünftigen Kapitalflüssen resp. Erträgen versprochen werden, sodass Eigenschaften von Aktien, Obligationen oder auch physische Gegenstände auf einer Blockchain gespeichert werden können. Wesentlicher Bestandteil dieser Eigenschaften ist der Eigentümer des Tokens. Die Blockchain übernimmt in diesen Fällen die Funktion einer Börse. Damit diese Art von Token genutzt werden kann, bedarf es in den meisten Fällen einer gesetzlichen Regelung. Im Sinne des Finanzmarktinfrastrukturgesetzes handelt es sich bei Anlage-Token um Effekte. 


\subsection{Einordnung der Kryptowährungen}

Die Frage, wie die Kryptowährungen einzuordnen sind, stellt sich bei der technischen Abbildung wie aber auch beim regulatorischen Ausweis. In vielen Bereichen ist diese Einordnung der Kryptowährungen zum aktuellen Zeitpunkt noch nicht klar.

Die Kryptowährungen werden im Vergleich zu normalen Währungen nicht von einem Staat ausgegeben. Daher wird der Wert auch nicht durch die Geldpolitik eines Landes beeinflusst. Als Währung im eigentlichen Sinn kann die Kryptowährung, auch ,virtuelle Währung“ genannt, nicht bezeichnet werden (Thiele und Diehl 2017). Stattdessen ist die Unterstellung, beispielsweise als Zahlungsmittel oder Anlageinstrument, bei den einzelnen Gesetzen separat zu prüfen (FINMA 2018; Zordi Bernkopf und Seiler 2019).

Geld erfüllt grundsätzlich drei Funktionen: Es dient als Zahlungsmittel, als Recheneinheit und als Wertaufbewahrungsmittel. Aus ökonomischer Sicht können die Kryptowährungen aufgrund der fehlenden generellen Akzeptanz aktuell (noch) nicht als Währungen bezeichnet werden. Dazu müsste die Kryptowährung als allgemeines Zahlungsmittel akzeptiert werden. Auch die Funktion als Recheneinheit ist umstritten, da aktuell die meisten Händler, welche Bitcoin akzeptieren, die Preise zum Transaktionszeitpunkt an die aktuellen Umrechnungskurse von Bitcoin anpassen. Die Berechnung der Preise erfolgt somit weiterhin mithilfe einer Fiat-Währung ${ }^{1}$ (Read und Gräslund 2018).

Da hinter der Kryptowährung im Gegensatz zu klassischen Anlagen kein Unternehmen steht, kann die Volatilität nicht mit sich ändernden Gewinnerwartungen an ein Unternehmen erklärt werden. Bei den Kryptowährungen handelt es sich ausschließlich um die Erwartung, dass in Zukunft der Kurs sich aufgrund anderer Marktteilnehmer mit positiven Erwartungen entsprechend entwickeln wird.

\subsubsection{Wichtige Begriffe}

Zentrale Begriffe des vorliegenden Beitrags werden jetzt kurz erörtert:

\section{Private Key}

Jeder Teilnehmer benötigt einen privaten Schlüssel. Der Schlüssel besteht aus einer Bitfolge, die als Zahlen und Buchstaben dargestellt werden kann. Der Schlüssel wird normalerweise in einem Wallet aufbewahrt und allenfalls auch durch die Wallet-Software generiert. Da die Kryptowährungen allein mit diesem privaten Schlüssel transferiert werden können, sollte dieser immer geheim bleiben (Seiler und Seiler 2018, S. 153).

\footnotetext{
${ }^{1}$ Beispiele: EUR, USD, CHF: Es handelt sich um eigentlich nutzlose Objekte, welche als Tauschmittel eingesetzt werden (Wallace 2019).
} 


\section{Wallet}

Ein Wallet ist eine Art digitaler Geldbeutel, in welchem die Kryptowährungen abgespeichert werden. Um Überweisungen aus einem Wallet zu tätigen, muss der Besitzer seinen privaten Schlüssel verwenden. Im Kontext der Kryptowährungen wird immer wieder von unterschiedlichen Wallet-Typen gesprochen. Die spezifischen Eigenschaften werden nachfolgend kurz erläutert (Dölle 2019; Lansky 2018).

\section{Cold-Wallet}

Als Cold-Wallet werden diejenigen bezeichnet, welche nicht aktiv für den Zahlungsverkehr verwendet werden. Man könnte diese vergleichen mit einem Sparkonto. Meistens werden Cold-Wallets eingerichtet, damit der größere Bestand des Kapitals sicherer verwahrt werden kann. Beispielsweise kann der entsprechende Private Key physisch auf einem Speichermedium vor Zugriff geschützt werden, etwa in einem sicheren Tresor.

\section{Hot Wallet}

Diese Wallets werden für den laufenden Zahlungsverkehr und die laufenden Transaktionen genutzt. Die Private Keys zu diesem Wallet sind beispielsweise auf einem Arbeitsplatzrechner, einem Server (bei kommerzieller Verwendung) oder sogar auf einem MobileDevice abgespeichert. Diese Geräte sind meistens mit dem Internet verbunden. Aufgrund der Speicherung auf aktiven Geräten besteht ein gewisses Risiko, dass ebendiese Geräte verloren gehen, gestohlen werden oder die Daten durch Cyberkriminalität abfließen.

\subsubsection{Bitcoin}

Bitcoin ist die verbreitetste Kryptowährung (Grundlehner et al. 2018) und die älteste. Sie weist die erste praktische Umsetzung einer Blockchain auf (Morgenschweis 2018). Im November 2018 wurden täglich weltweit zwischen 240.000 und 280.000 Transaktionen durchgeführt (Dölle 2018). Damit ist die Kapazität von rund sieben Transaktionen pro Sekunde etwa zur Hälfte ausgeschöpft (Hanl und Michaelis 2017, S. 364). Im Vergleich zu den gut 1,7 Mio. Schweizer Transaktionen pro Tag des SIC-Systems (SIX Interbank Clearing) ist die Bedeutung für den Zahlungsverkehr noch gering (SIC-Statistiken - SIX 2018).

Die Volatilität des Bitcoins ist sehr hoch, verglichen mit anderen traditionellen Anlageinstrumenten, wie der Abb. 7.2 zu entnehmen ist. Allein im Jahr 2019 hat sich der Wert des Bitcoins gemessen in US\$ per 15. Mai 2019 verdoppelt. Interessant ist die Eigenschaft, dass die Kryptowährungen keine Korrelation mit den Marktschwankungen anderer Anlagen zeigen. Trotzdem besteht jederzeit das Risiko eines Totalverlusts, da kein Gegenwert vorhanden ist (Fiedler et al. 2018).

Das Marktvolumen wird bekanntlich ab 2024 konstant sein, da die festgelegte Anzahl Blocks zu diesem Zeitpunkt erreicht sein wird. Damit wird der Bestand an Bitcoin stabiler als Gold (jährliches Wachstum zwischen 1,5 und $2 \%$ ) (Thole 2019). 


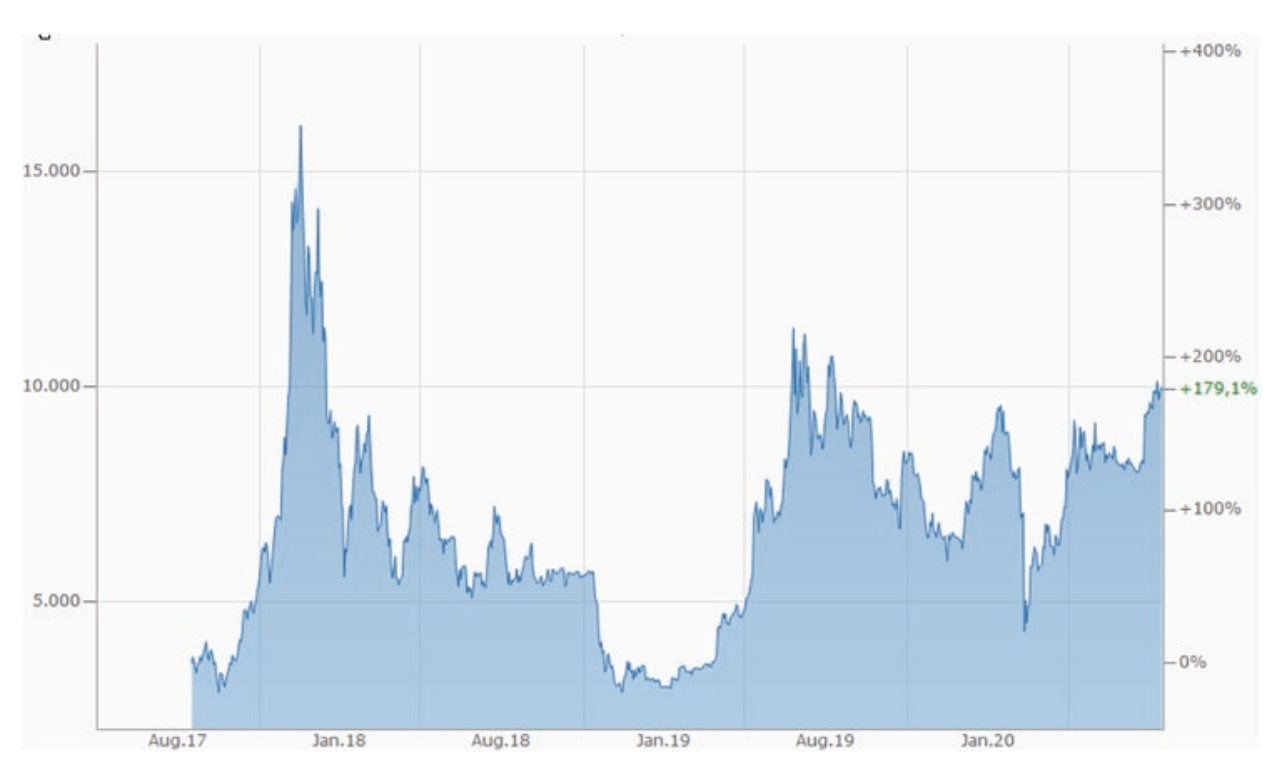

Abb. 7.2 Chart Bitcoin/US-Dollar. (Quelle: Cash 2020)

\subsection{Kryptowährungen im Bankenumfeld}

Zu den Kryptowährungen sind im Bankenumfeld aktuell keine verbreiteten Anwendungen bekannt. Bekannt ist, dass verschiedene Banken Projekte durchführen mit dem Ziel, Kryptowährungen in ihren Banksystemen zu implementieren. Dabei wurden bereits erste Transaktionen durchgeführt, Angebote zu Dienstleistungen im Markt sind aber noch selten.

\subsubsection{Standpunkte aktueller Softwareanbieter}

Bankensoftware, wie z. B. Finnova haben schon erste Umsetzungen bekannt gegeben, welche die Implementierung von Kryptowährungen ermöglichen. Viele Projekte laufen mit verschiedenen Banken, welche die Implementierung von Kryptowährungen als Ziel haben.

Die Implementierungen haben aktuell zwei Ansätze. Zum einen werden Kryptowährungen als Valor in den Depots abgebildet und es werden Kryptowährungen in Kontoform geführt. Favorisiert wird aktuell die Umsetzung als Valor, insbesondere wenn es sich um öffentliche Kryptowährungen handelt und nicht um von der Bank selbst emittierte Coins (Finnova AG Bankware 2019).

Neben der Kernbankensoftware wird zusätzliche Software für die Abbildung der Kryptowährungen benötigt. FinTechs beschäftigen sich auch mit der Distributed Ledger Technologie, welche die Basis der Blockchain bildet. Entsprechend sollte es relativ einfach 
möglich sein, hier entsprechende Anbieter zu finden (Ankenbran et al. 2019; Ankenbrand 2019).

\subsubsection{Die Nachkommastellen im Bankenumfeld}

In den bestehenden Banksystemen werden die Fiat-Währungen mit zwei Nachkommastellen geführt (Rappen bei CHF, Cent bei EUR). Aus technischer Sicht existiert zudem in jedem Datenfeld eine Begrenzung, welche die minimale und maximale Zahl sowie die Genauigkeit begrenzen. Bei Kryptowährungen wird in vielen Fällen mit wesentlich mehr Nachkommastellen gearbeitet. Für die Kryptowährung Bitcoin z. B. gibt es acht Nachkommastellen, sodass bei der Umsetzung der Kryptowährungen für jedes einzelne System und jedes einzelne Datenfeld die Frage gestellt werden muss, ob die Anzahl verfügbarer Nachkommastellen ausreicht. Da Rundungen in den Kontoführungen nicht erlaubt sind, sind Anpassungen in diesen Bereichen zwingend notwendig.

Eine Alternative besteht darin, in den Systemen jeweils eine kleinere Einheit als die Basiseinheit abzubilden. Dies müsste entsprechend spezifiziert werden. Hierbei liegt die Herausforderung darin, dass die Einheit bei jeder Schnittstelle genau spezifiziert und umgesetzt wird. Es ist davon auszugehen, dass Systeme, welche mit unterschiedlichen Einheiten arbeiten, zu technischen Problemen führen und für den Benutzer kaum nachvollziehbar sein werden (Bradbury 2013) (Tab. 7.1).

\subsection{Einsatzmöglichkeiten von Kryptowährungen}

\subsubsection{Einsatz im Zahlungsverkehr}

Der Fachbereich Zahlen (siehe Abb. 7.1) beschäftigt sich mit der Infrastruktur für den Zahlungsverkehr. Der ursprüngliche Einsatzzweck des Bitcoins war der Zahlungsverkehr, welches auch das erste Einsatzszenario darstellt. Aufgrund der unterschiedlichen Konkurrenzsysteme im inländischen und ausländischen Zahlungsverkehr werden die Einsatzmöglichkeiten separat aufgeführt. Aus Sicht der Kryptowährung spielen die Landesgrenzen aber keine Rolle.

Tab. 7.1 Einheiten von Kryptowährungen

\begin{tabular}{l|l|l}
\hline Währung & Kleinste Einheit & Bezeichnung \\
\hline CHF & 0,01 & Rappen \\
\hline EUR & 0,01 & Cent \\
\hline Ripple & 0,000001 & Drop \\
\hline Bitcoin & 0,00000001 & Satoshi \\
\hline Ethereum & 0,000000000000000001 & Wei \\
\hline $\begin{array}{l}\text { Quellen: bitcoinmag.de 2017; Bradbury 2013; } \\
\text { Ethereum-kaufen.de 2019 }\end{array}$
\end{tabular}




\subsubsection{Nationaler Zahlungsverkehr Schweiz}

Im nationalen Zahlungsverkehr haben sich in der Praxis bisher keine nennenswerten Anwendungsmöglichkeiten gezeigt, da die Kryptowährung im Vergleich zu bestehenden Zahlungsmöglichkeiten keine bedeutenden Vorteile bieten. Tab. 7.2 soll die Anwendungsmöglichkeiten von Kryptowährungen im nationalen Zahlungsverkehr aufzeigen und bereits bestehende Alternativen des bisherigen Banksystems gegenübergestellt werden.

Einziger Use Case, welcher aktuell durch bisherige Zahlungssysteme nicht abgedeckt werden kann, ist die Eliminierung des Intermediären. Diese Eliminierung hat für die meisten Kunden vermutlich keinen wesentlichen Nutzen. Insbesondere in der Schweiz, wo das Vertrauen in den Staat und in die Banken sehr hoch ist (Brunner 2017), besteht für die meisten Kunden kaum ein Grund, nur dafür auf Kryptowährungen als Zahlungsmittel auszuweichen.

Insbesondere im Zusammenhang mit der Digitalisierung und den damit verbundenen mobilen Bezahllösungen (oli/sda 2019) wird aber der Schritt zum mobilen Kryptowallet ein wesentliches Stück kleiner. Für die Technologie wäre es ein großer Schritt, wenn einer der großen digitalen Marktplayer auf Kryptowährungen setzen würde.

\subsubsection{Internationaler Zahlungsverkehr}

Im internationalen Zahlungsverkehr nimmt die Komplexität der Zahlungen insbesondere dann wesentlich zu, wenn die Zahlung den SEPA²-Raum verlässt. Mit dem Einbezug von

Tab. 7.2 Kryptowährung im nationalen Zahlungsverkehr

\begin{tabular}{|c|c|c|}
\hline Use-Case & Vorteil Kryptowährung & Alternative \\
\hline $\begin{array}{l}\text { Zahlung am Point } \\
\text { of Sale }(\mathrm{PoS})^{\mathrm{a}}\end{array}$ & $\begin{array}{l}\text { Direkte Bezahlung mit } \\
\text { Smartphone möglich }\end{array}$ & $\begin{array}{l}\text { Kontaktloses bezahlen mit Kredit- und } \\
\text { Debitkarten }\left(\mathrm{NFC}^{\mathrm{b}}\right) \text { oder Twint }{ }^{\mathrm{c}}\end{array}$ \\
\hline $\begin{array}{l}\text { Bezahlung von } \\
\text { Rechnungen }\end{array}$ & $\begin{array}{l}\text { Direkte Freigabe am } \\
\text { Computer oder mobil }\end{array}$ & $\begin{array}{l}\text { Erfassung der Zahlung im E-Banking/Mobile } \\
\text { Banking }^{\mathrm{d}} \text { oder Nutzung von eBille und } \\
\text { Lastschriftverfahren (LSV) }\end{array}$ \\
\hline $\begin{array}{l}\text { Überweisung an } \\
\text { Bankkonto }\end{array}$ & $\begin{array}{l}\text { Direkte Freigabe am } \\
\text { Computer oder mobil }\end{array}$ & $\begin{array}{l}\text { Erfassung der Zahlung im Banking/Mobile } \\
\text { Banking }\end{array}$ \\
\hline $\begin{array}{l}\text { Automatisierte } \\
\text { Bezahlung }\end{array}$ & $\begin{array}{l}\text { Software kann Zahlung } \\
\text { automatisch tätigen }\end{array}$ & $\begin{array}{l}\text { Zahlung kann via eBill oder LSV automatisch } \\
\text { freigegeben werden }\end{array}$ \\
\hline $\begin{array}{l}\text { Reduktion der } \\
\text { Gebühren }\end{array}$ & Geringe Transaktionskosten & $\begin{array}{l}\text { Je nach Zahlungsart geringe Gebühren, } \\
\text { werden teilweise aber pauschal bezahlt }\end{array}$ \\
\hline $\begin{array}{l}\text { Eliminierung des } \\
\text { Intermediär }\end{array}$ & $\begin{array}{l}\text { Transaktionen ohne } \\
\text { Banken/Clearingstelle } \\
\text { möglich }\end{array}$ & Keine Alternative \\
\hline
\end{tabular}

Quelle: eigene Darstellung

${ }^{a}$ Verkaufs- oder Einkaufsstelle, abhängig von der Perspektive, die man betrachtet

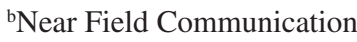

'Twint 2019

${ }^{\mathrm{d} A u s f u ̈ h r u n g ~ v o n ~ B a n k g e s c h a ̈ f t e n ~ m i t ~ e i n e m ~ m o b i l e n ~ G e r a ̈ t ~(S m a r t p h o n e ~ o d e r ~ T a b l e t) ~}$

${ }^{\mathrm{e}}$ Mit eBill erhalten Kunden ihre Rechnungen direkt auf ihr E-Banking-Konto

\footnotetext{
${ }^{2}$ Single European Payment Area.
} 
Tab. 7.3 Kryptowährung im internationalen Zahlungsverkehr

\begin{tabular}{l|l|l}
\hline Use Case & Vorteil Kryptowährung & Alternative \\
\hline $\begin{array}{l}\text { Zahlung am Point of } \\
\text { Sale (PoS) }\end{array}$ & $\begin{array}{l}\text { Direkte Bezahlung mit Smartphone } \\
\text { möglich }\end{array}$ & $\begin{array}{l}\text { Kontaktloses Bezahlen mit } \\
\text { Kredit- und Debitkarten resp. NFC }\end{array}$ \\
\hline $\begin{array}{l}\text { Bezahlung von } \\
\text { Rechnungen }\end{array}$ & $\begin{array}{l}\text { Direkte Freigabe am Computer oder } \\
\text { mobil }\end{array}$ & $\begin{array}{l}\text { Erfassung der Zahlung im } \\
\text { Banking/Mobile Banking }\end{array}$ \\
\hline $\begin{array}{l}\text { Überweisung an } \\
\text { Bankkonto }\end{array}$ & $\begin{array}{l}\text { Direkte Freigabe am Computer oder } \\
\text { Mobil }\end{array}$ & $\begin{array}{l}\text { Erfassung der Zahlung im } \\
\text { Banking/Mobile Banking }\end{array}$ \\
\hline $\begin{array}{l}\text { Automatisierte } \\
\text { Bezahlung }\end{array}$ & $\begin{array}{l}\text { Software kann Zahlung automatisch } \\
\text { tätigen }\end{array}$ & Keine Alternative \\
\hline $\begin{array}{l}\text { Reduktion der } \\
\text { Gebühren }\end{array}$ & $\begin{array}{l}\text { Transaktionen kosten geringe } \\
\text { Gebühren, steigen nicht im } \\
\text { internationalen Verkehr }\end{array}$ & $\begin{array}{l}\text { Internationale Zahlungen sind } \\
\text { teurer als die Zahlung in } \\
\text { Kryptowährung }\end{array}$ \\
\hline $\begin{array}{l}\text { Eliminierung des } \\
\text { Intermediär }\end{array}$ & $\begin{array}{l}\text { Transaktionen sind ohne Banken/ } \\
\text { Clearingstelle möglich }\end{array}$ & Keine Alternative \\
\hline Rasche Zahlung & $\begin{array}{l}\text { Zahlung innerhalb von wenigen } \\
\text { Minuten möglich }\end{array}$ & Zahlungen dauern mehrere Tage \\
\hline
\end{tabular}

Quelle: eigene Darstellung

Korrespondenzbanken ${ }^{3}$ und somit auch wesentlich höheren Aufwänden steigen auch die Gebühren auf Seiten der Kunden (Tab. 7.3).

Im Vergleich zum nationalen Zahlungsverkehr bestehen weitere Vorteile. Namentlich die tiefen Gebühren und einfachere Möglichkeiten für die Automatisierung von Zahlungen. Somit hätte der Einsatz von Kryptowährungen für den internationalen Zahlungsverkehr Vorteile gegenüber den bestehenden Alternativen (Brühl 2017, S. 141).

Maßgebende Schwierigkeiten, welche im internationalen Zahlungsverkehr durch Kryptowährungen überwunden werden könnten, sind Vorgaben wie FATCA, ${ }^{4}$ die Abhängigkeiten zu Korrespondenzbanken und Unsicherheiten bezüglich des Erfolgs und der Dauer der Transaktion (Arnold und Knödler 2018, S. 423).

\subsubsection{Begrenzende Faktoren für Kryptowährungen als Zahlungsmittel}

Als begrenzende Faktoren für den Einsatz von Kryptowährungen können zum aktuellen Zeitpunkt mehrere Bereiche genannt werden, welche sich primär auf die am weitesten verbreitete Kryptowährung Bitcoin beziehen. Je nach Ausgestaltung einer anderen Kryptowährung können diese Faktoren weniger zutreffen oder sogar vollständig eliminiert werden (Tab. 7.4).

\footnotetext{
${ }^{3}$ Bank in einem anderen Land, mit der eine Geschäftsbeziehung unterhalten wird.

${ }^{4}$ Foreign Account Tax Compliance Act.
} 
Tab. 7.4 Begrenzende Faktoren

\begin{tabular}{l|l}
\hline Gebühren & $\begin{array}{l}\text { Um die Aufwände der Minera finanzieren zu können, werden in } \\
\text { Zukunft die Transaktionsgebühren ansteigen. Dadurch wird sich } \\
\text { der aktuelle Bonus der günstigen Transaktionsgebühren im } \\
\text { Vergleich zu herkömmlichen Zahlungssystemen weiter reduzieren. }\end{array}$ \\
\hline Anzahl & $\begin{array}{l}\text { Die Anzahl der Transaktionen, welche die Kryptowährungen } \\
\text { innerhalb eines Blocks verarbeiten können, sind begrenzt. Die } \\
\text { entsprechende Begrenzung könnte zwar durch Anpassungen der } \\
\text { Regeln angehoben werden. Dazu benötigt es aber eine Mehrheit } \\
\text { der Teilnehmer, welche die Anpassung übernehmen. }\end{array}$ \\
\hline Transaktionsgeschwindigkeit & $\begin{array}{l}\text { Die Verarbeitungszeit einer Transaktion ist an die Verarbeitung } \\
\text { eines Blocks in einer bestimmten Zeitspanne gebunden. Wenn die }\end{array}$ \\
& $\begin{array}{l}\text { Transaktion aufgrund vieler konkurrierender Transaktionen nicht } \\
\text { direkt in den nächsten Block aufgenommen wird, kann es auch } \\
\text { noch länger dauern, bis diese verarbeitet wird. Insbesondere am } \\
\text { Point of Sale (PoS) wäre aber eine umgehende Verarbeitung } \\
\text { notwendig, um die Kryptowährung einzusetzen. }\end{array}$ \\
\hline
\end{tabular}

${ }^{a}$ Miner verarbeiten die Transaktionen der Kryptowährungen zu Blöcken

\subsubsection{Einsatz als Anlagevehikel}

Die Kryptowährungen werden von verschiedenen Experten als alternative Anlagemöglichkeit zu bestehenden Anlageinstrumenten angesehen. Gerade weil die Entwicklung der Kryptowährungen nicht mit anderen Anlageinstrumenten korreliert, kann eine entsprechende Investition das bestehende Portfolio diversifizieren. Aufgrund der hohen Volatilität handelt es sich um eine tendenziell spekulative Anlage. Mit einem Investment in Kryptowährungen setzt man aber nicht auf das Wachstumspotenzial eines Unternehmens, sondern spiegelt die Meinung des Anlegers wider, dass die Blockchain-Technologie in der Zukunft an Bedeutung gewinnen wird.

\subsubsection{Finanzieren}

Das Hauptgeschäft der Regionalbanken, die Kreditvermittlung, wird im Fachbereich Finanzieren unterstützt. Die Abwicklung der Kreditanträge über die Bewilligung bis zur Auszahlung, aber auch die anschließende Abbildung in den Systemen mit der Zins- und Amortisationsberechnung und Belastung wird in diesem Fachbereich abgebildet. Dazu zählt auch die Erfassung der entsprechenden Deckungen, wie zum Beispiel der Grundstücke mit den diversen Eigenschaften. Auch die Meldungen an die Pfandbriefbank und das Überzugsmanagement wird im Fachbereich Finanzieren betreut. 


\subsubsection{Einsatz im Kreditwesen}

Die Kryptowährungen können im Kreditbereich ebenfalls eingesetzt werden. Wie bei anderen Fremdwährungsfinanzierungen ist dies besonders für Kunden interessant, welche auch sonst Einnahmen oder Auslagen in der entsprechenden Kryptowährung haben. Im Gegensatz zu Krediten in Währungen wie Euro würde sich der Kredit in einer Kryptowährung nicht primär auf Grenzregionen fokussieren.

Im Rahmen der Bitcoin-Finanzierungen werden insbesondere auch direkte Kapitalvermittlungen ohne Beteiligung einer Bank möglich. In diesen Konstellationen stellt sich die Frage, ob die Bank allenfalls Dienstleistungen in Form von Kreditprüfungen anbieten kann. Die Anleger könnten dann auf Basis der von der Bank ermittelten Daten ihren Anlageentscheid treffen und so ihr Vermögen direkt beim Kreditnehmer anlegen.

\subsection{Rahmenbedingungen von Kryptowährungen}

Damit die Kryptowährungen verbreitet zum Einsatz kommen, müssen verschiedene Rahmenbedingungen erfüllt sein. In Tab. 7.5 werden die Rahmenbedingungen erläutert, welche für den breiten Einsatz von Kryptowährungen bei Regionalbanken erfüllt sein müssen.

\subsection{Adaption Modellbank}

Mit den gewonnenen Erkenntnissen zu den Einsatzmöglichkeiten und deren Rahmenbedingungen wird klar, welche Bereiche der Modellbank von allfälligen Änderungen betroffen sind. Bei den beschriebenen Use Cases ist in den meisten Fällen softwareseitige Unterstützung unabdingbar. Indem die neuen Prozesse mit der bestehenden Softwarelandschaft verglichen werden, wird ermittelt bei welchen Fachbereichen der Modellbank Software und/oder Prozessanpassungen notwendig sind. Nachfolgend werden verschiedene mögliche Dienstleistungsangebote dargestellt, welche Einfluss auf die Modellbank haben.

\subsubsection{Kryptowährungen als Asset}

Der Fachbereich Anlegen wird durch Kryptowährungen in erster Linie tangiert. Eine mögliche Erweiterung des Dienstleistungsangebots der Regionalbanken ist das Aufnehmen der Kryptowährungen als zusätzliche Asset-Kategorie. Neben den bisherigen Asset-Kategorien kann neu Kryptowährung als zusätzliche Kategorie aufgenommen werden, um die Portfoliodiversifikation weiter voranzutreiben. Hierbei ist zu unterscheiden zwischen direkten und indirekten Investments in dieser Asset-Kategorie. 
Tab. 7.5 Rahmenbedingungen

\begin{tabular}{|c|c|}
\hline Rechtliche & $\begin{array}{l}\text { Erste Aufsichtsmitteilungen der FINMA zur Anwendung von } \\
\text { Kryptowährungen im Zusammenhang mit Geldwascherei und } \\
\text { Zahlungsverkehr wurde seit } 2019 \text { veröffentlicht (FINMA 2019). Da es sich } \\
\text { bei Kryptowährungen nicht um Währungen im Sinne der geläufigen } \\
\text { Definition handelt, stellt sich in verschiedensten Belangen die Frage, wie die } \\
\text { Kryptowährungen genau zu behandeln sind. Damit die Kryptowährungen } \\
\text { verbreitet zum Einsatz kommen können, ist es sinnvoll, entsprechende Regeln } \\
\text { zu verfassen, damit der Umgang einheitlich wird. } \\
\text { Rechtliches Risiko: In verschiedenen Bereichen besteht das Risiko bei } \\
\text { einer Implementierung von Kryptowährungen, dass die rechtlichen } \\
\text { Anforderungen einer anderen Implementierung bedürfen. } \\
\text { Je nach Implementierung der Kryptowährungen sind unterschiedliche } \\
\text { rechtliche Regelungen notwendig. Um das Investitionsrisiko zu reduzieren, } \\
\text { sind allenfalls neue Regelungen abzuwarten. } \\
\text { Die Schweiz setzt nicht auf ein eigenes Blockchain-Gesetz, sondern passt } \\
\text { bestehende Gesetze an die neuen Bedürfnisse an. Die ersten Ergebnisse aus } \\
\text { entsprechenden Analysen gingen bereits im März } 2019 \text { in die } \\
\text { Vernehmlassung (Maurer 2019). }\end{array}$ \\
\hline Technologische & $\begin{array}{l}\text { Zusätzliche Softwarebestandteile sind einzukaufen oder zu programmieren. } \\
\text { Hierbei bestehen bereits Angebote von Drittherstellern, welche mittels } \\
\text { Schnittstellen an die bestehenden Systeme angebunden werden können. } \\
\text { Die Abbildung der vielen Nachkommastellen von verschiedenen } \\
\text { Kryptowährungen ist zu lösen. Andere Komponenten wie die technische } \\
\text { Anbindung, Internetanbindung und Anforderungen an Server und } \\
\text { Speichersysteme sind vielmehr eine Frage der Organisation als eine } \\
\text { technologische Herausforderung. }\end{array}$ \\
\hline Kundenorientierte & $\begin{array}{l}\text { Nach Einschätzung der Autoren haben die meisten Kunden zum aktuellen } \\
\text { Zeitpunkt kein effektives Bedürfnis nach Kryptowährungen, da sie im } \\
\text { Alterssegment über } 40 \text { Jahre kaum vorhanden sind. Aus heutiger Sicht ist bei } \\
\text { keiner Einsatzmöglichkeit der Vorteil für die Kunden groß genug, dass sie } \\
\text { bereit wären, die zusätzlichen Risiken zu akzeptieren. }\end{array}$ \\
\hline Interne & $\begin{array}{l}\text { Aktuell haben die Mitarbeiter der Regionalbanken wenig Know-how im } \\
\text { Bereich der Kryptowährungen. Damit die Dienstleistungen erfolgreich sein } \\
\text { können, müssen die Mitarbeiter zuerst geschult werden. } \\
\text { Damit Dienstleistungen mit Kryptowährungen erfolgreich bei Kunden } \\
\text { platziert werden können, ist es unerlässlich, ein Schulungsprogramm für die } \\
\text { Mitarbeitenden aufzuziehen. }\end{array}$ \\
\hline Marktbedingte & $\begin{array}{l}\text { Aktuell beschäftigen sich verschiedene Banken mit der Einführung von } \\
\text { Kryptowährungen. Mit den Veränderungen der Dienstleistungen rund um } \\
\text { Kryptowährungen steigt auch der Druck auf den Regulator an, Vorgaben zu } \\
\text { veröffentlichen, damit die Marktangebote bereits früh an gewisse Regeln } \\
\text { gebunden sind. }\end{array}$ \\
\hline
\end{tabular}




\section{Indirekte Investments}

Mit Investments in bestehende Anlageinstrumente wie Anlagefonds oder Derivate kann der Anleger an der Kursentwicklung von Kryptowährung partizipieren. Es bestehen schon Angebote an strukturierten Produkten. Beispielsweise hat Vontobel schon im Oktober 2017 ein Partizipationszertifikat auf Bitcoin herausgegeben (Vontobel 2017). Bei diesen Anlageinstrumenten ist es auch denkbar, mehrere Kryptowährungen als Basiswert zu hinterlegen, um die Produktrendite zu erhöhen oder um die Abhängigkeit zum Bitcoin zu reduzieren. Problematisch bei solchen strukturierten Produkten ist nach wie vor das Emittentenrisiko.

Mittels Futures-Kontrakte kann der Kunde direkt auf die Kursentwicklung von Bitcoin spekulieren und sowohl ,long“- wie ,short“-Positionen eingehen. Futures auf der CME Börse ${ }^{5}$ haben eine Kontraktgröße von fünf Bitcoins und Laufzeiten für sechs aufeinanderfolgende Monate und zwei zusätzliche im Dezember.

\section{Direkte Investments}

Kunden investieren direkt in Kryptowährungen, welche die Bank für sie im Depot aufbewahrt. Der Kunde kann sich somit im Anlagegespräch neben den klassischen Anlageinstrumenten auch für einen Anteil Kryptowährungen entscheiden. Die Bank würde in diesem Fall die Kryptowährung in ihrem System als synthetischen Valor führen. Die Abbildung würde somit wie bei herkömmlichen Wertschriften erfolgen, ohne dass eine Wertschrift vorliegt. Dieser wird dem Kunden beim Kauf ins Depot eingebucht. Damit hat der Kunde auf seinem Depotauszug eine zusätzliche Position, welche die Kryptowährungen zeigt.

\subsubsection{Kryptowährungen als Zahlungsmittel}

Die Umsetzung eines Dienstleistungsangebots für den Zahlungsverkehr ist zu berücksichtigen, obwohl Kryptowährungen so designt sind, dass jede Person selbst ein Wallet ohne Finanzintermediäre erstellen kann. Das Dienstleistungsangebot im Bereich Zahlungsverkehr müsste aus Sicht der Autoren die Verwaltung des Wallets als Kern haben.

\section{Zentrale Verwaltung von Wallet}

Ein mögliches Setup für die Dienstleistung mit einer zentralen Verwaltung von Wallets wird in der Abb. 7.3 gezeigt.

Mit der zentralen Verwaltung von Wallets hat der Kunde den Vorteil, dass er seinen privaten Key nicht selbst sicher verwahren muss. Zudem können Konstellationen mit Todesfällen und Erteilung von Vollmachten vermutlich einfacher gelöst werden. Zur Sicherheit können, zumindest bei höheren Beständen, mehrere Wallets geführt werden. Die Kunden hätten somit ein Konto mit einer besonderen Währung. Für Überweisungen mit Kryptowährungen müsste ein neuer Typ Zahlungsauftrag erstellt werden. Überweisungen

${ }^{5} \mathrm{CME}$ group https://www.cmegroup.com/trading/equity-index/us-index/bitcoin.html. 


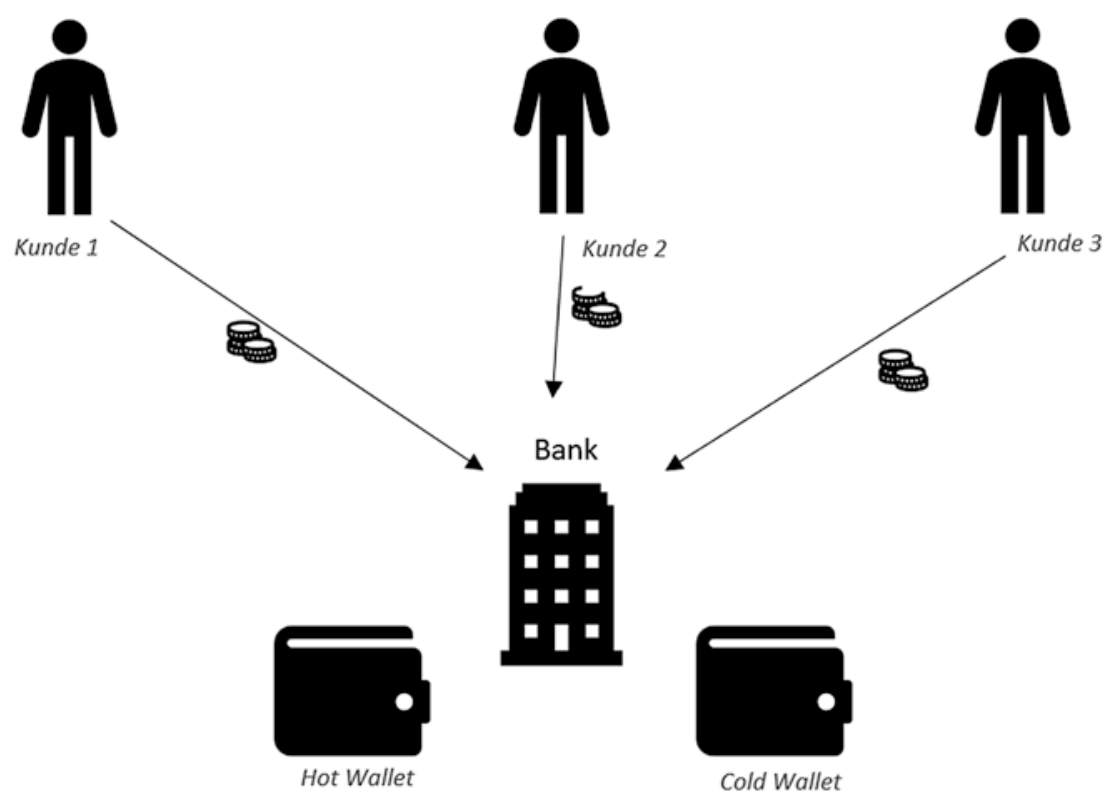

Abb. 7.3 Setup Dienstleistungen Zahlungsverkehr mit zentralem Wallet. Die Guthaben der Kunden werden in Wallets der Bank zusammengelegt

in Kryptowährungen an andere Personen würde die Bank dann aus ihrem Wallet ausführen. Für die Überweisung an den Kunden kann beispielsweise eine bestimmte Mitteilung verlangt werden, oder es werden individuelle Adressen für die Überweisungen erstellt. Nur mit einer dieser Optionen wäre es möglich, die Transaktionen einem bestimmten Kunden zuzuordnen. Die eingehenden Beträge müssen somit anschließend durch die Bank dem entsprechenden Konto des Kunden zugeordnet werden. Der Kunde kann der Bank zudem den Auftrag geben, Kryptowährungen zu kaufen oder zu verkaufen. Entsprechend würde die Bank die Kryptowährungen dem Kunden auf dem Kryptokonto gutschreiben/ belasten und entsprechend den Gegenwert dem Privatkonto des Kunden belasten/gutschreiben.

Das zentrale Wallet bedeutet aber auch, dass die Bank ebendieses Wallet sehr gut schützen muss. Zudem kann in der Blockchain jeder nachvollziehen, welches Wallet welche Transaktionen ausgeführt hat. Hier muss geprüft werden, ob eine Identifikation zu verhindern ist, weil es problematisch sein könnte, wenn die Öffentlichkeit die Adressen der Wallets kennt. Diese erhöhte Transparenz ist aber mit der Gefahr verbunden, dass vertrauliche Bankdaten an die Öffentlichkeit gelangen könnten.

\section{Dezentrale Verwaltung: ein Wallet je Kunde}

Ein anderer Ansatz wäre, für jeden Kunden ein eigenes Wallet zu führen. Damit würde die Problematik der Identifizierung umgangen. Zudem wäre es so für den Kunden einfacher, entsprechende Transaktionen zu empfangen, da er einfach seine individuelle Adresse bekannt geben kann. 
Beim Kauf und Verkauf von Kryptowährungen kann die Bank den Gegenwert dem Privatkonto des Kunden belasten und die Transaktion an das individuelle Wallet des Kunden auslösen und umgekehrt.

Bei der Konstellation mit individuellen Wallets wären die Krypto-Konten der einzelnen Kunden vermutlich nur ein Abbild des entsprechenden Wallets. Entsprechend ist bei dieser Konstellation auch der rechtliche Zusammenhang zu prüfen. Gehört in dieser Konstellation dem Kunden direkt das Wallet? Besteht somit ein Besitzanspruch und nicht eine Forderung gegenüber der Bank? Dies würde einer vollständigen Deckung entsprechen. Entsprechend kann die Bank die Einlagen des Kunden auch nicht für weitere Zwecke nutzen, wie dies bei Sparkonten möglich wäre (Abb. 7.4).

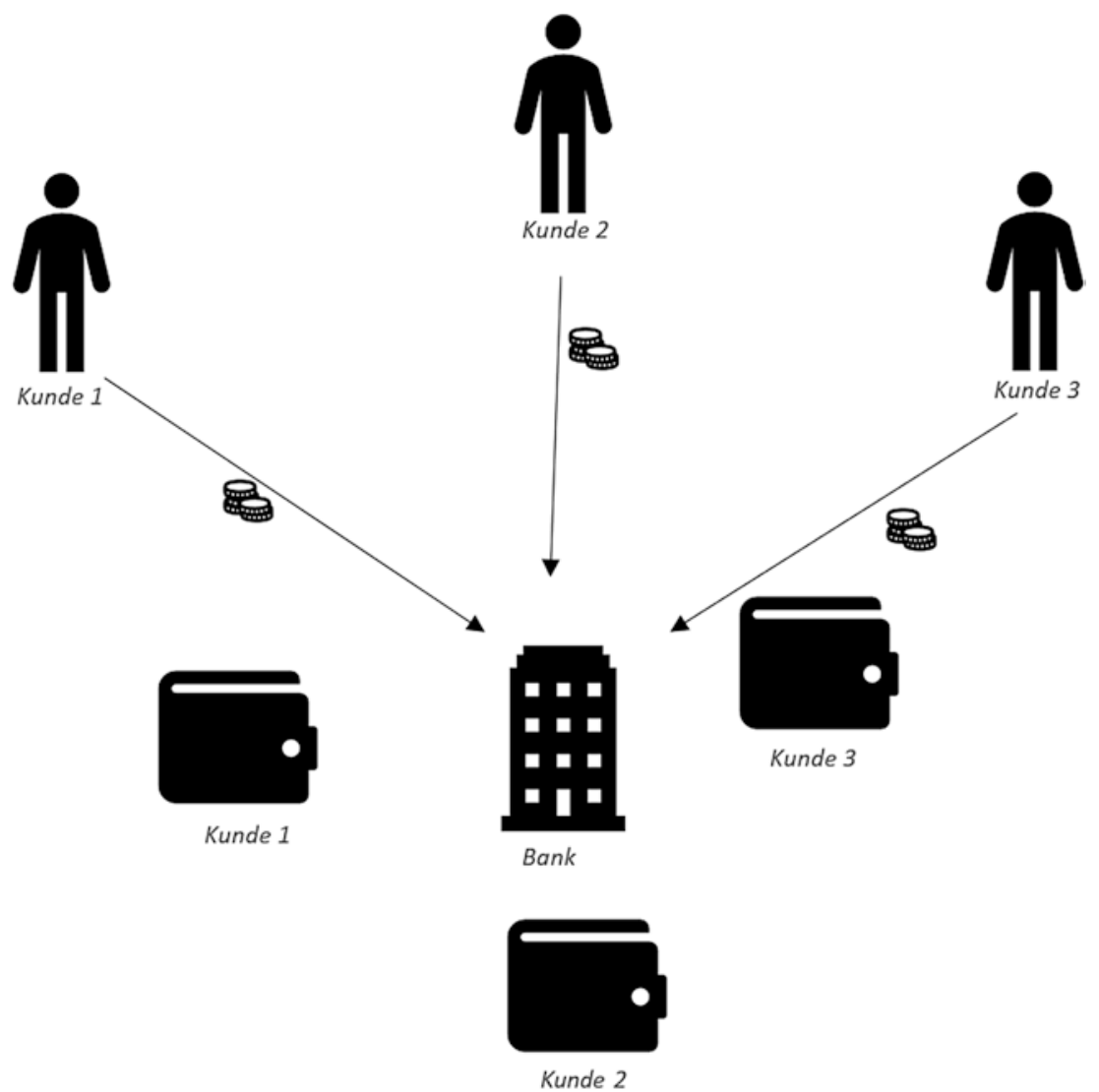

Abb. 7.4 Setup Dienstleistungen Zahlungsverkehr mit einzelnen Wallets. Für jeden Kunden wird ein (oder mehrere) separates Wallet geführt 


\subsubsection{Begleitung von Initial Coin Offerings}

Im Rahmen der Kapitalbeschaffung von KMU wäre es denkbar, als KMU selbst AnlagenToken herauszugeben (Abschn. 7.3), sodass sie interessierte Personen in ihrem Umfeld auf diese Weise eine Beteiligung ermöglichen würden.

Die Aufgabe der Banken wäre hier die fachliche Begleitung der entsprechenden Kapitalbeschaffungen und eine effiziente Durchsetzung zu gewährleisten. Neben der Beratung sind in der Begleitung weitere Dienstleistungen, wie das technische Aufsetzen, das Berechnen der kryptografischen Token sowie Dienstleistungen in den Bereichen Storage und Brokerage möglich. Der Nutzen gegenüber alternativen Finanzierungsmöglichkeiten sollte in tieferen Kosten liegen und eine neue Anlagemöglichkeit für kleine Investoren darstellen.

\subsubsection{Kredite in Kryptowährungen}

Insbesondere Firmenkunden, welche vermehrt Einnahmen in Kryptowährungen erwarten, könnten Interesse an einer entsprechenden Finanzierung haben. Da sie mit den Einnahmen in Kryptowährungen ihre Kredite amortisieren könnten, müssten sie die Kryptowährungen nicht in CHF für die Amortisation zurücktauschen. Die Auszahlung des Kreditbetrags zum Beginn der Laufzeit kann je nach Produktspezifikation und nach Kundenbedürfnissen in einer Kryptowährung oder im entsprechenden Gegenwert in CHF erfolgen.

Die Finanzierung durch die Bank könnte nahezu identisch wie bei Finanzierungen mit Kryptowährungen ablaufen. Bei der Prüfung der Kreditfähigkeit wäre aber zu berücksichtigen, dass entsprechende Geldflüsse in Kryptowährungen vorliegen müssten. Andernfalls würde ein theoretisch unbegrenztes Risiko in der Kurssteigerung der entsprechenden Kryptowährung liegen.

\subsection{Make- or Buy-Entscheidung}

Unabhängig der in Frage kommenden Dienstleistungsangebote muss ein strategischer Entscheid erfolgen, welchen Teil der Dienstleistung die Bank selbst leistet und welchen Teil sie von einer Drittpartei einkaufen will. Entsprechend wären die Anforderungen an die eigenen Ressourcen wesentlich unterschiedlich. Betroffen von entsprechenden Überlegungen müssen insbesondere die Software für Storage und Brokerage der Kryptowährungen sein. Die Komponente Storage kümmert sich um die Speicherung der Kryptowährungen (Wallet-Funktion) und verwaltet mehrere Wallets für unterschiedliche Kunden. Die Komponente Brokerage ermöglicht den Kauf und Verkauf von Kryptowährungen bei einer entsprechenden Börse als Broker. Die Überlegungen der nachfolgenden Kapitel beziehen sich auf Tab. 7.6. 
Tab. 7.6 Vorteile und Nachteile

\begin{tabular}{c|l|l}
\hline & Make-Entscheidung & Buy-Entscheidung \\
\hline Vorteile & Aufbau von vertieftem Know-how zu & Schnellere und kostengünstige \\
& Kryptowährungen durch selbstständige & Implementierung, da bereits erste \\
& Umsetzung entsprechender Storage- und & Lösungsangebote auf dem Markt \\
verfügbar sind. & Rrokerage-Lösungen. & Reduktion des Investitionsrisikos. \\
& Erbringung neuer Dienstleistungen in & \\
& Krytopwährungen bringt wesentlichen & \\
& Mehrnutzen und Diversifikation. & \\
& Erhöhtes Wachstum durch & \\
& Differenzierungsstrategie in diesem Bereich & \\
\hline Nachteile & Höhere Investitionskosten, da entsprechende & Operationelles Risiko durch starke \\
& Fachkräfte rekrutiert werden müssen. & Abhängigkeiten zum neuen \\
& Hohes Investitionsrisiko, da zum aktuellen & Geschäftspartner. \\
& Zeitpunkt nicht abgeschätzt werden kann, \\
& welchen Umsatz die entsprechenden & Reputationsrisiko, falls sich der \\
& Dienstleistungsangebote je erzielen werden. & Geschäftspartner nicht korrekt \\
& verhalten würde. \\
& Hohe Wechselkosten, falls die künftige & Entstehung von Verlustrisiko im \\
& Marktentwicklung nicht den aktuellen & Betrugsfall. \\
& Marktanforderungen entsprechen würde. & \\
\hline
\end{tabular}

\subsection{Zusammenfassung der Ergebnisse}

Die Implementierung von Kryptowährungen in die Modellbank weist verschiedene Ausprägungen auf und derer Umsetzung sollte ein breiteres Dienstleistungsangebot ermöglichen. Die identifizierten, potenziellen Dienstleistungen wurden in Abschn. 7.8.1 detailliert erläutert:

- Kryptowährung als Asset: Die verschiedenen Kryptowährungen können zur Wertanlage dienen.

- Kryptowährung als Zahlungsmittel: digitaler Austausch zum Bezug von Waren und Dienstleistungen.

- Begleitung von ICO: Kapitalbeschaffung für kleine und mittelgroße Unternehmen.

- Kredite in Kryptowährungen: Finanzierungen in digitalen Währungen für Kunden mit Erträgen in Kryptowährungen.

Je nach gewünschtem Dienstleistungsangebot sind unterschiedliche Handlungsgebiete zu bearbeiten, wobei die Schwierigkeit in der Unsicherheit liegt, da der Markt mit Dienstleistungen in Kryptowährungen noch relativ jung ist.

Aufgrund der identifizierten Handlungsfelder kann abgeschätzt werden, wie entsprechende Projekte zur Implementierung aufgesetzt werden können. Der Umfang der Projekte muss zusammen mit den entsprechenden Stakeholdern definiert werden sowie die Umsetzung einer Kauflösung, welche wesentlich schneller umsetzbar wäre. 
Die Abbildung von Kryptowährungen als Asset wäre aus heutiger Sicht die am schnellsten umsetzbare Lösung. Für die Abbildung von Nutzungs- und Anlagetoken würde diese Lösung die meisten Ansprüche aus heutiger Sicht abdecken.

\section{Kritische Würdigung}

Um die Nachfrage der Kunden nach entsprechenden Lösungen zu ermitteln, wäre eine groß angelegte Umfrage nötig. Alternativ und mit geringeren Kosten verbunden wäre eine Umfrage bei sämtlichen Frontmitarbeitern, die näher am Markt sind und dementsprechend die Kundenanforderungen besser einschätzen können.

Um einen besseren Eindruck von den bestehenden Lösungen zu erhalten, wäre zudem ein Ghost-Shopping sinnvoll, indem die Kontaktaufnahme als Kunde bei anderen Banken erfolgt. Dabei können sowohl interne als auch externe Personen beauftragt werden, um zu erforschen, welche Lösungen andere Anbieter aktuell haben. Das Ghost-Shopping kann aber nur da angewandt werden, wo ein Angebot für den Retail-Markt existiert.

Um Dienstleistungsangebote und Handlungsfelder besser zu spezifizieren, wäre es sinnvoll, Kontakt mit entsprechenden Dienstleistungsanbietern aufzunehmen. Je nach Partnerunternehmen können sich die Details der Dienstleistung sowie die zu bearbeitenden Handlungsfelder noch leicht verschieben.

\subsection{Fazit}

Die Umsetzung der Kryptowährungen für die Modellbank hat bei den meisten Szenarien wesentlichen Anpassungsbedarf zur Folge.

In Abb. 7.5 werden die wichtigsten Anpassungen für die jeweiligen Dienstleistungsangebote zusammengefasst.

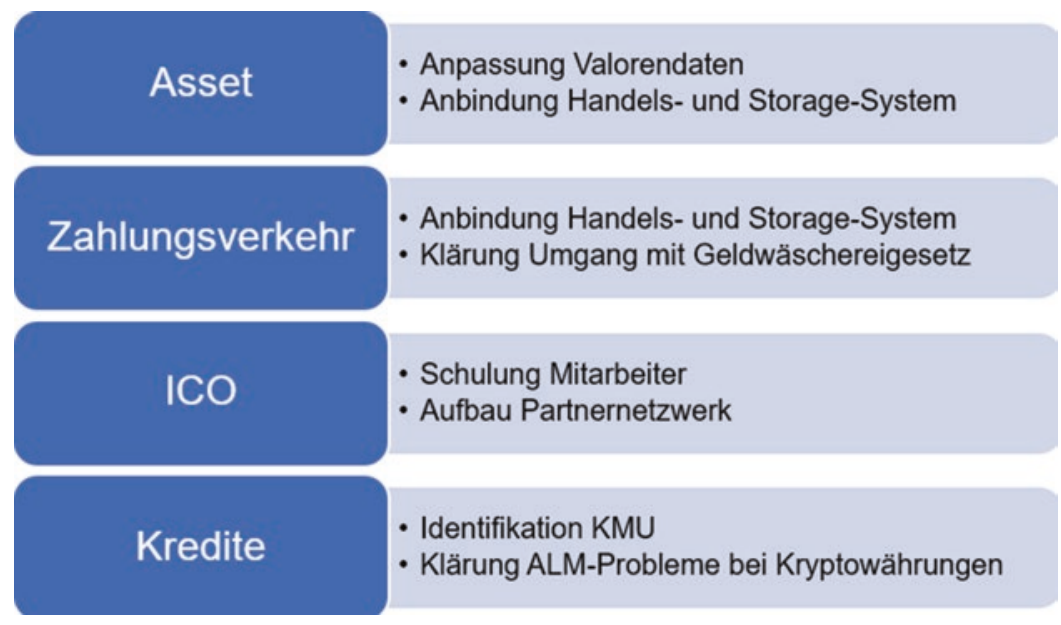

Abb. 7.5 Wichtigste Anpassungen für Dienstleistungsangebote. (Quelle: eigene Darstellung) 
Die betroffenen Bereiche der Modellbank differieren je nach Dienstleistungsangebot wie auch der Anpassungsumfang. Die Umsetzung der Kryptowährungen muss auf jeden Fall in einem bereichsübergreifenden Projekt erfolgen, bei welcher die verschiedenen Business Analysten der jeweiligen Fachbereiche involviert sind.

Je nach Handlungsoptionen bei der Umsetzung der verschiedenen Dienstleistungen sind die Ausprägungen als Make- or Buy-Variante immer möglich.

Da die Regulatorien betreffend Kryptowährungen noch nicht sehr ausgereift und die Anbindungen an die Kernbankensoftware noch nicht definiert sind, ist die favorisierte Handlungsoption, aktuell keine Dienstleistungen umzusetzen. Trotzdem sollte ein Monitoring aufgebaut werden, um die Entwicklungen im Bereich der Kryptowährungen aktiv zu verfolgen. Zudem sollten beim Monitoring auch die weiteren Anwendungsgebiete der Blockchain-Technologie eingebunden werden, da diese viel Potenzial zugesprochen wird.

Die Blockchain-Technologie sollte auf jedem Fall durch ein institutionalisiertes Gefäß aktiv verfolgt werden.

Die Herausforderung besteht darin, das Marktpotenzial richtig einzuschätzen und ob bestehenden Kunden Bedürfnisse an solchen Produkten haben. Dazu wäre es sinnvoll ein Monitoring aufzubauen, damit entsprechende Anfragen von Kunden konsequent registriert werden. Die Datenbank kann später auch zur Unterstützung des CRM ${ }^{6}$ dienen, wenn solche Dienstleistungen eingeführt werden.

Das Monitoring der Entwicklungen auf Angebotsseite als auch der permanente Kontakt mit Lösungsanbietern ist unentbehrlich. Im Falle einer Einführung könnte die entsprechende Konzeptionierung der Lösung wesentlich rascher erfolgen, wenn bereits Beziehungen zu potenziellen Partnern existieren.

Zum aktuellen Zeitpunkt ist es sicherlich ratsam, auf die Regulatorien abzuwarten, da noch einige neue Regeln im Umgang zu Kryptowährungen publiziert werden können.

Zugunsten einer „Early-Follower-Strategie“ sollten die ersten Abklärungen und Konzepte für eine rasche Umsetzungen getroffen werden.

\section{Literatur}

Ankenbran, T., Dietrich, A., \& Bieri, D. (2019). IFZ FinTech study 2019. An overview of Swiss FinTech. Lucerne: Institute of Financial Services Zug IFZ.

Ankenbrand, T. (2019). Finnova Expert Panel Investment Management. Wie können Banken die FinTech Evolution überleben? (April 2019). Lenzburg: Finnova AG Bankware.

Arnold, C., \& Knödler, H. (Hrsg.). (2018). Die informatisierte Service-Ökonomie. Veränderungen im privaten und öffentlichen Sektor. Wiesbaden: Springer Gabler.

Bieri, N., \& Uhlmann, K. (2019). Technologieneutralität als Richtschnur im Umgang mit der Blockchain. In Eidgenössisches Departement für Wirtschaft, Bildung und Forschung (Hrsg.), Block-

\footnotetext{
${ }^{6} \mathrm{CRM}$ ist ein Tool zur systematischen Gestaltung der Kundenbeziehungsprozesse und beinhaltet alle zur Kunden relevanten Informationen und Interaktionen zwischen Bank und Kunde.
} 
chain: Mehr als ein Hype? Die Volkswirtschaft (Bd. 92(5), S. 9-11 [Themenheft]). Bern: Staatssekretariat für Wirtschaft SECO.

bitcoinmag.de. (2017). Was ist Ripple (XRP)?|Bitcoin-WikilbitcoinMag.de. https://www.bitcoinmag.de/bitcoin-wiki/was-ist-ripple-xrp. Zugegriffen am 08.06.2019.

Bradbury, D. (2013). The problem with Bitcoin. Computer Fraud \& Security, 2013(11), 5-8. https:// doi.org/10.1016/S1361-3723(13)70101-5.

Brühl, V. (2017). Bitcoins, Blockchain und Distributed Ledgers. Wirtschaftsdienst, 97(2), 135-142. https://doi.org/10.1007/s10273-017-2096-3.

Brunner, S. (2017). Sorgenbarometer 2017: Das Vertrauen der Schweizer in ihre Institutionen (Credit Suisse, Hrsg.). https://www.credit-suisse.com/corporate/de/articles/news-and-expertise/ worry-barometer-2017-the-swiss-place-high-trust-in-their-institutions-201712.html. Zugegriffen am 21.05.2019.

Cash. (Hrsg.). (2020). Bitcoin Kurs in US Dollar (BTC/USD). https://www.cash.ch/devisen-zinsen/ bitcoin-in-usd-39475121/nexc/usd. Zugegriffen am 25.04.2020.

Dölle, M. (2018). So richtig falsch. Warum eine vielzitierte Klim-Studie zu Bitcoin falsch liegt. C't Magazin für Computer und Technik (26), 30.

Dölle, M. (2019). Todsicher. Tausende Bitcoins als Grabbeigabe. c't - Magazin für Computer und Technik 2019(6), 50-51. https://www.heise.de/select/ct/2019/6/1552648097706426. Zugegriffen am 03.05.2019.

Ethereum-kaufen.de. (2019). Ethereum Einheiten. https://ethereum-kaufen.de/ethereum-einheiten/. Zugegriffen am 08.06.2019.

Fiedler, S., Gern, K.-J., \& Stolzenburg, U. (2018). Kryptowährungen - Geld der Zukunft? Wirtschaftsdienst, 98(10), 752-754. https://doi.org/10.1007/s10273-018-2362-z.

FINMA, E. F. (16. Februar 2018). Wegleitung. für Unterstellungsfragen betreffend Initial Coin Offerings (ICOs). https://www.finma.ch/de/news/2018/02/20180216-mm-ico-wegleitung/. Zugegriffen am 10.05.2019.

FINMA, E. F. (Hrsg.). (2019). Laufende Anhörungen der FINMA. https://www.finma.ch/de/dokumentation/anhoerungen/laufende-anhoerungen/. Zugegriffen am 14.05.2019.

Finnova AG Bankware. (2019). Insight 19. April 2019 (Finnova AG Bankware, Hrsg.). Lenzburg: Finnova AG Bankware.

Grundlehner, W., Schürpf, T., \& Bauer, D. (2018). Bitcoin \& Co.: Kryptowährungen im Überblick. NZZ Neue Zürcher Zeitung AG, Schweiz. https://www.nzz.ch/finanzen/bitcoinkryptowaehrungen-im-ueberblick-ld.1336477. Zugegriffen am 05.01.2019.

Gussmann, A. (2018). Initial Coin Offerings. Tokens im Kontext der Shared Economy (BearingPoint, Hrsg.). Amsterdam: BearingPoint.

Hanl, A., \& Michaelis, J. (2017). Kryptowährungen - ein Problem für die Geldpolitik? Wirtschaftsdienst, 97(5), 363-370. https://doi.org/10.1007/s10273-017-2145-y.

Lansky, J. (2018). Possible state approaches to cryptocurrencies. Journal of Systems Integration, 9(1), 19-31. https://doi.org/10.20470/jsi.v9i1.335.

Maurer, U. (2019). Die Schweiz ist eine der führenden Nationen im Bereich Fintech und Blockchain. In Eidgenössisches Departement für Wirtschaft, Bildung und Forschung (Hrsg.), Blockchain: Mehr als ein Hype? Die Volkswirtschaft (Bd. 92 (5), S. 5 [Themenheft]). Bern: Staatssekretariat für Wirtschaft SECO.

McMillan, J. (2018). Das Ende der Banken. warum wir sie nicht brauchen (1. Aufl.). Frankfurt a. M.: Campus.

Morgenschweis, S. (2018). Initial Coin offerings und Kryptowährungen. Die Bank, (4), 65-67.

oli/sda. (2019). Google macht Twint Konkurrenz, Tamedia Espace AG ((Berner Zeitung, Hrsg.)). https://www.bernerzeitung.ch/24456542. Zugegriffen am 03.05.2019.

Read, O., \& Gräslund, K. (2018). EU-Regulierung von Bitcoin und anderen virtuellen Währungen: erste Schritte. Wirtschaftsdienst, (7), 504-511. 
Seiler, B., \& Seiler, D. (2018). Sind Kryptowährungen wie Bitcoin (BTC), Ethereum (ETH) und Ripple (XRP) als Sachen im Sinne des ZGB zu behandeln? sui generis. https://doi.org/10.21257/sg.65.

SIC-Statistiken - SIX. (2018). Transaktionen pro Monat. https://www.six-group.com/interbankclearing/de/home/profil/statistics/sic/trx-months.html. Zugegriffen am 05.01.2019.

Thiele, C.-L., \& Diehl, M. (2017). Kryptowährung Bitcoin: Währungswettbewerb oder Spekulationsobjekt. Welche Konsequenzen sind für das aktuelle Gelsystem zu erwarten? ifo schnelldienst, 70(22), 3-6.

Thole, F. (2019). Kryptowährungen: Gekommen, um zu bleiben. Die Bank, (4), 56-61.

Twint AG. (2019). die innovative Finanzdienstleisterin. Twint AG. https://www.twint.ch/ueber-uns/. Zugegriffen am 08.06.2019.

Vontobel. (Hrsg.). (2017). Neues Partizipationszertifikat auf Bitcoin mit unbegrenzter Laufzeit. https://zertifikate.vontobel.com/DE/blog/Artikel/neues-partizipationszertifikat-auf-bitcoin-mitunbegrenzter-laufzeit. Zugegriffen am 31.05.2019.

Wallace, N. (2019). Fiat money. In Palgrave Macmillan (Hrsg.), The new Palgrave dictionary of economics (Living reference work, Living edition, Bd. 91, S. 1-9). London: Palgrave Macmillan.

Zordi Bernkopf, S. de, \& Seiler, D. (2019). Regulierung von Krypto-Assets in der Schweiz (BDO Schweiz, Hrsg.). https://www.bdo.ch/de-ch/themen/fachartikel/banking-newsletter/regulierungvon-krypto-assets-in-der-schweiz. Zugegriffen am 22.05.2019.

Michael Mathys Masterstudium in Betriebswirtschaft an der Berner Fachhochschule mit Vertiefung Corporate/Business Development. Langjährige Berufserfahrung im Regionalbankenumfeld. Aktuell Business Analyst Enterprise Management bei der Dienstleisterin für Regionalbanken Clientis AG.

Raul Gimeno Professor für Banking \& Finance am Departement Wirtschaft der Berner Fachhochschule. Lehr- und Forschungstätigkeit in den Bereichen Asset Management, Investitionsrechnung und Unternehmensbewertung. Davor 13 Jahre Berufserfahrung im Bankenbereich.

Open Access Dieses Kapitel wird unter der Creative Commons Namensnennung 4.0 International Lizenz (http://creativecommons.org/licenses/by/4.0/deed.de) veröffentlicht, welche die Nutzung, Vervielfältigung, Bearbeitung, Verbreitung und Wiedergabe in jeglichem Medium und Format erlaubt, sofern Sie den/die ursprünglichen Autor(en) und die Quelle ordnungsgemäß nennen, einen Link zur Creative Commons Lizenz beifügen und angeben, ob Änderungen vorgenommen wurden.

Die in diesem Kapitel enthaltenen Bilder und sonstiges Drittmaterial unterliegen ebenfalls der genannten Creative Commons Lizenz, sofern sich aus der Abbildungslegende nichts anderes ergibt. Sofern das betreffende Material nicht unter der genannten Creative Commons Lizenz steht und die betreffende Handlung nicht nach gesetzlichen Vorschriften erlaubt ist, ist für die oben aufgeführten Weiterverwendungen des Materials die Einwilligung des jeweiligen Rechteinhabers einzuholen.

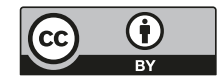

